\title{
Two-Level Anterior Lumbar Interbody Fusion With Percutaneous Pedicle Screw Fixation: A Minimum 3-Year Follow-up Study
}

\author{
Dong Yeob LEE, Sang-Ho LEE, and Dae Hyeon MAENG* \\ Departments of Neurosurgery and ${ }^{*}$ Thoracic and Cardiovascular Surgery, \\ Wooridul Spine Hospital, Seoul, R.O.K.
}

\begin{abstract}
The clinical and radiological outcomes of two-level anterior lumbar interbody fusion (ALIF) with percutaneous pedicle screw fixation (PSF) were evaluated in 24 consecutive patients who underwent twolevel ALIF with percutaneous PSF for segmental instability and were followed up for more than 3 years. Clinical outcomes were assessed using a visual analogue scale (VAS) score and the Oswestry Disability Index (ODI). Sagittal alignment, bone union, and adjacent segment degeneration (ASD) were assessed using radiography and magnetic resonance imaging. The mean age of the patients at the time of operation was 56.3 years (range 39-70 years). Minor complications occurred in 2 patients in the perioperative period. At a mean follow-up duration of 39.4 months (range 36-42 months), VAS scores for back pain and leg pain, and ODI score decreased significantly (from 6.5, 6.8, and $46.9 \%$ to $3.0,1.9$, and $16.3 \%$, respectively). Clinical success was achieved in 22 of the 24 patients. The mean segmental lordosis, whole lumbar lordosis, and sacral tilt significantly increased after surgery (from $25.1^{\circ}, 39.2^{\circ}$, and $32.6^{\circ}$ to $32.9^{\circ}, 44.5^{\circ}$, and $36.6^{\circ}$, respectively). Solid fusion was achieved in 21 patients. ASD was found in 8 of the 24 patients. No patient underwent revision surgery due to nonunion or ASD. Two-level ALIF with percutaneous PSF yielded satisfactory clinical and radiological outcomes and could be a useful alternative to posterior fusion surgery.
\end{abstract}

Key words: anterior lumbar interbody fusion, lumbar vertebra

\section{Introduction}

The well-known drawbacks of posterior lumbar fusion surgery are extensive injuries to the paraspinal muscles, which are thought to contribute to so-called "fusion disease," destruction or damage to the posterior midline complexes, neurological deficits, radiculitis, and others. ${ }^{8,11,14,26)}$ Anterior lumbar interbody fusion (ALIF) could be a solution to these problems related to posterior fusion surgeries, because the anterior retroperitoneal exposure used to access the disk space avoids stripping of the lumbar paraspinal muscles, preserves the posterior midline complexes, and minimizes the risks of neural complications. ${ }^{4,10,14,19,22)}$ ALIF with percutaneous pedicle screw fixation (PSF) is supposed to be a good alternative for posterior lumbar fusion surgery, especially in patients requiring multi-level fusion surgery. However, no study has specifically focused on the

Received November 17, 2009; Accepted March 17, 2010 surgical outcomes of two-level ALIF with percutaneous PSF.

The present study evaluated the clinical and radiological outcomes of patients who underwent twolevel ALIF with percutaneous PSF for segmental instability and were followed up for more than 3 years.

\section{Materials and Methods}

A total of 132 patients underwent ALIF with percutaneous PSF for segmental instability, single level surgery in 108 patients and two-level surgery in 24 patients, between September 2003 and August 2004. A retrospective review was performed of the 24 patients, 8 males and 16 females aged 39-70 years (mean 56.3 years), who underwent two-level ALIF with percutaneous PSF for segmental instability. All patients presented with low back pain, leg pain, and/or claudication, persisting for 2-72 months (mean 17.2 months). The inclusion criteria of this study were as follows: adjacent two-level lumbar pathol- 
ogies, i.e., segmental instability on radiography, computed tomography, and/or magnetic resonance (MR) imaging; radicular symptoms, claudication, and/or back pain consistent with the radiologic findings; and unsuccessful conservative therapy administered for at least 6 weeks. Radiographic criteria for segmental instability were as follows: sagittal plane displacement greater than $4.5 \mathrm{~mm}$ or $15 \%$ of the anteroposterior diameter of the vertebral body on standing lateral radiography, relative sagittal angulation greater than 22 degrees on standing lateral radiography, sagittal plane translation greater than $4.5 \mathrm{~mm}$ or $15 \%$ of the anteroposterior diameter of the vertebral body on dynamic radiography, or sagittal plane rotation greater than 20 degrees on dynamic radiography. ${ }^{6)}$ Patients with previous history of spinal surgery, traumatic lumbar fracture, tumor, or infection were excluded. Patients with segmental instability combined with central stenosis, which required simultaneous posterior decompression, were also excluded.

Before and after surgery, pain was measured by the 10-point visual analogue scale (VAS) scoring, and functional status was assessed by the Oswestry Disability Index (ODI) scoring. ${ }^{5)}$ Clinical success was defined as follows: $\geq 2$ point improvement in VAS score, $\geq 25 \%$ improvement in ODI score, and no major complication related to the surgery. ${ }^{15,27)}$ Radiography and MR imaging were performed before and 3 years after surgery to assess sagittal alignment, bone union, and adjacent segment degeneration (ASD).

To evaluate changes in sagittal balance after the interbody fusion, the preoperative and postoperative (at last follow up) segmental lordosis of the fused segment, whole lumbar lordosis, and sacral tilt were assessed using standing lateral radiography. Segmental lordosis at L3-L4-L5 was defined as the angle subtended by the superior endplate line of L3 and inferior endplate line of L5. Segmental lordosis at L4L5-S1 was defined as the angle subtended by the superior endplate line of $\mathrm{L} 4$ and the endplate line of S1. Whole lumbar lordosis was defined as the angle subtended by the superior endplate line of L1 and the endplate line of S1. Sacral tilt was defined as the angle subtended by the endplate line of $\mathrm{S} 1$ and a horizontal reference line. Bone union was assessed using standing lateral and flexion-extension lateral radiography at the final follow up. The criteria of bone union were as follows: presence of trabecular osseous bridge, less than $4^{\circ}$ mobility between segments on static and dynamic lumbar radiographs, and absence of lucency around the interbody cage. The criteria of nonunion were as follows: visible gap, graft collapse, and/or motion greater than $4^{\circ}{ }^{\circ}{ }^{21)}$
A patient was defined to have ASD if radiography and MR imaging showed one or more of the following lesions adjacent to the fused segment, which was not present preoperatively: loss of disk height of more than $10 \%$, anterolisthesis or retrolisthesis greater than $4 \mathrm{~mm}$, angle change greater than $10^{\circ}$ between adjacent vertebral bodies on flexion and extension radiographs, occurrence of symptomatic disk herniation or spinal stenosis, hypertrophic facet joint arthropathy, osteophyte greater than 3 $\mathrm{mm}$, scoliosis, and compression fracture. ${ }^{15,20,21)}$

Statistical analysis was performed using the paired sample $t$ test. A $\mathrm{p}$ value less than 0.05 was considered significant.

All operations followed a standard pattern, with all procedures supervised by the senior surgeon. ${ }^{17)}$ The retroperitoneal exposure was performed by a vascular surgeon in all patients. The patient was placed in the supine position under general anesthesia. The retroperitoneal approach was performed through a midline skin incision. The anterior longitudinal ligament and anterior annulus were removed after careful dissection and retraction of the common iliac artery and vein. Disk removal and preparation of the end plate was then performed. After removing the posterior annulus using a Kerrison punch, anterior decompression of nerve root in the foramen was performed until the posterior longitudinal ligament had been exposed. Then, decompression of the nerve roots in the bilateral foramina was confirmed with probing. Appropriate anterior decompression was indicated by the free passage of the probe into the bilateral foramina on fluoroscopic imaging. Lordotic cages, which had a lordosis of $12^{\circ}$, were used in all patients for interbody fusion. The cages filled with graft material (cancellous allograft) were placed into the disk spaces. The patient was then placed into the prone position for supplemental percutaneous PSF. After making bilateral paraspinal incisions, bilateral pedicle screw-rod placement was accomplished under the guidance of C-arm fluoroscopy via the bilateral paraspinal routes.

\section{Results}

All patients underwent surgery for two-level segmental instability. Twenty-two of the 24 patients had spondylolisthesis (two-level spondylolisthesis in 9 and one-level spondylolisthesis in 13). The affected levels were L3-L4-L5 in 5 patients and L4-L5-S1 in 19 patients. The mean operation time was 252.5 minutes (range 165-390 minutes). The mean estimated blood loss was $478.1 \mathrm{~cm}^{3}$ (range $250-850 \mathrm{~cm}^{3}$ ). Transfusion was performed in two cases. All 

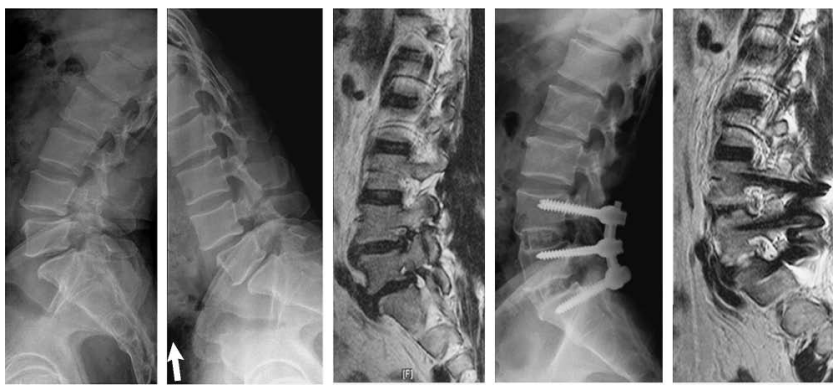

Fig. 1 Preoperative dynamic radiograms (left and left center) and sagittal $T_{2}$-weighted magnetic resonance image (center) showing foraminal stenosis and spondylolysis combined with segmental instability at the L4-L5 and L5-S1 levels. White arrow means that dynamic radiograms are taken in standing posture. Radiogram (right center) and sagittal $T_{2}$-weighted magnetic resonance image (right) taken 3 years after surgery demonstrating solid fusion and successful decompression of foraminal stenosis.

patients showed improvement of symptoms related to radiculopathy immediately after surgery. There was no major morbidity or mortality in the perioperative period. There were two minor complications in the perioperative period; one case of ileus and one of wound dehiscence. Both cases were successfully treated without any sequelae. Twenty patients started to ambulate at 1 or 2 days after surgery. Four patients showed delay in ambulation due to postoperative abdominal and/or back pain (three patients at 3 days after surgery and one patient at 4 days after surgery). The mean hospital stay was 8.6 days (range 5-24 days).

The mean follow-up duration was 39.4 months (range 36-42 months). The mean preoperative VAS score for back pain was 6.5 (range 2-10) and improved after surgery to 3.0 (range $0-4)(p<0.001)$. The mean preoperative VAS score for leg pain was 6.8 (range 3-9) and improved after surgery to 1.9 (range $0-4)(\mathrm{p}<0.001)$. The mean preoperative ODI score was $46.9 \%$ (range $24.4-71.1 \%$ ) and improved after surgery to $16.3 \%$ (range $4.4-42.2 \%$ ) ( $<<0.001$ ). Clinical success was seen in 22 of the 24 patients (Figs. 1 and 2).

The mean preoperative segmental lordosis was $25.1^{\circ}$ (range $-9.9-42.6^{\circ}$ ) and significantly increased after surgery to $32.9^{\circ}$ (range $4.1-57.1^{\circ}$ ) ( $p<0.001$ ). The mean preoperative whole lumbar lordosis was $39.2^{\circ}$ (range $8.1-61.1^{\circ}$ ) and significantly increased after surgery to $44.5^{\circ}$ (range $\left.17.0-79.1^{\circ}\right)(\mathrm{p}=0.028$ ). The mean preoperative sacral tilt was $32.6^{\circ}$ (range $\left.15.1-55.8^{\circ}\right)$ and significantly increased after surgery to $36.6^{\circ}$ (range $\left.16.3-51.9^{\circ}\right)(\mathrm{p}=0.002)$. The intraobserver reliability for radiological measurements was

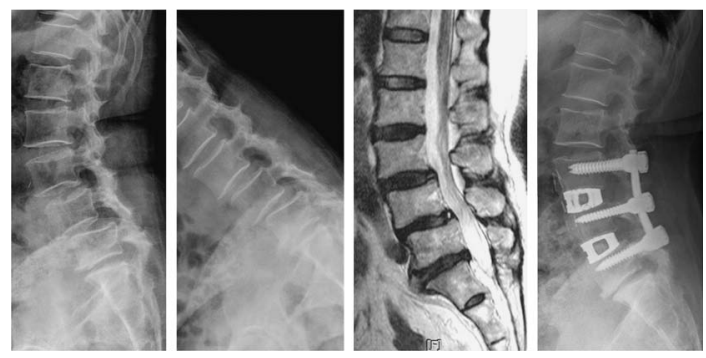

Fig. 2 Preoperative radiograms (left and left center) and sagittal $T_{2}$-weighted magnetic resonance image (right center) showing degenerative spondylolisthesis combined with segmental instability at L3-L4 and L4-L5 level. Radiogram (right) taken 3 years after surgery demonstrating solid fusion.

0.92. Radiological evidence of solid fusion was observed in 21 of the 24 patients. Three patients were considered to have nonunion, two patients showed screw fracture during the follow-up period, which was managed with conservative treatment. Reoperation was not performed in any of the 3 cases of nonunion, and the outcome was successful in all these 3 patients despite the non-union. ASD was found in 8 of the 24 patients. ASD was found at the cranial segment in all 8 patients. Seven patients showed loss of disk height (more than 10\%) and one patient showed angular instability at the cranial segment. None of these 8 patients had clinical symptoms related to ASD. No patient underwent revision surgery due to non-union or ASD.

\section{Discussion}

Since the introduction of ALIF to treat spondylolisthesis, the clinical results of ALIF for degenerative lumbar diseases have been favorable and comparable to those of posterior fusion surger-

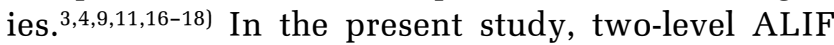
with percutaneous PSF yielded good clinical results; the mean values of VAS (for back pain and leg pain) and ODI scores decreased significantly after surgery and clinical success was achieved in 22 of the 24 patients. The good clinical results of the present study might be attributable to the advantages of ALIF over posterior fusion surgery, as decompression and fusion can be achieved without removing the posterior bony structures and retracting the neural structures. ${ }^{14,16)}$ In ALIF, anterior removal of the herniated disk decompresses the nerve roots and thecal sac. Then disk height elevation and slippage reduction enlarge the neural foramen, which results in additional decompression of the nerve root in the neuroforamen. ${ }^{16)}$ Patients with spondylolisthesis are 
more likely to experience reduced postoperative pain and improved functional status than patients with degenerative disk disease after ALIF. ${ }^{4,19)}$ Therefore, the high proportion of our patients (22 of 24) with spondylolisthesis seems another reason for good clinical results of the present study.

Posterior lumbar interbody fusion (PLIF) can produce circumferential decompression of the neural tissue as well as a biomechanically stable postoperative spine, but PLIF has a high perioperative complication rate. ${ }^{7,24,26)}$ PLIF resulted in 91 complications in $75(51 \%)$ of 148 patients. $^{24)}$ Perioperative complications occurred in $90(37.5 \%)$ of 240 patients who underwent PLIF with a high incidence of neurological complications (24.5\%). ${ }^{7}$ The relative risk of a perioperative complication is 4.75 times higher for PLIF than for ALIF. ${ }^{26)}$ In the present study, the postoperative complication rate was relatively low $(2 / 24,8.3 \%)$ in the perioperative period and all complications were regarded minor. No neurological complication occurred. Anterior approaches do carry the risk of catastrophic injuries to major vessels and other retroperitoneal and intraperitoneal structures. ${ }^{13)}$ In our series, an experienced vascular surgeon performed the anterior exposure in all patients and no approach-related major complication occurred.

Lumbar interbody fusion immediately establishes a biomechanically stable postoperative spine, and thus enhances the opportunity for arthrodesis. ${ }^{28)}$ Previously reported fusion rates after PLIF ranged from $56 \%$ to $100 \%,{ }^{2,12,23)}$ and fusion rates after ALIF ranged from $71 \%$ to $100 \%{ }^{4,9,10,18,20,25)}$ In multi-level fusion surgery, the fusion rate of two-level ALIF with percutaneous PSF is acceptable $(87.5 \%)$ and comparable to that of single level surgery. None of our patients with non-union underwent revision surgery. The wide fusion bed provided by the anterior approach and posterior pedicle screw augmentation might explain the relatively good fusion rate of the present study.

ALIF may have an advantage over PLIF in preventing the development of ASD. ${ }^{20)}$ Comparison of single-level instrumented ALIF with instrumented PLIF found ASD in $44 \%$ of the patients in the ALIF group and $82.6 \%$ of the patients in the PLIF group. ${ }^{20)}$ In the present study, we used the same criteria for the definition of ASD as that study. ${ }^{20)}$ In the present study, the incidence of ASD after twolevel fusion surgery was low; radiological evidence of ASD was found in 8 of 24 patients (33.3\%) and no patients underwent revision surgery due to ASD. Reduced damage to the integrity of the posterior complex during ALIF with percutaneous PSF, which is an important risk factor for ASD, might ex- plain the relatively low incidence of ASD in the present study. ${ }^{28)}$ Sagittal balance is believed to be responsible for the long-term outcome of patients undergoing fusion procedures, especially with regard to ASD. ${ }^{1,28)}$ Hypolordotic alignment at L4-L5 causes the greatest amount of flexion-extension motion at L3-L4, and the differences are statistically significant compared with in situ or hyperlordotic fixation. ${ }^{1)}$ Hypolordosis in the instrumented segments causes increased load on the posterior column of the adjacent segments, which might explain the degenerative changes at the junctional level that have been observed as long-term consequences of lumbar fusion. ${ }^{28)}$ Therefore, significant increase of segmental lordosis, whole lumbar lordosis, and sacral tilt after two-level ALIF with percutaneous PSF is another reason for the low incidence of ASD in the present study.

In conclusion, two-level ALIF with percutaneous PSF yielded satisfactory clinical and radiological outcomes and could be a useful alternative to posterior fusion surgery. However, the retrospective study design and relatively small number of patients enrolled should be considered when interpreting the results of the present study.

\section{Acknowledgment}

This study was supported by a grant from the Wooridul Spine Foundation.

\section{References}

1) Akamaru T, Kawahara N, Tim Yoon S, Minamide A, Su Kim K, Tomita K, Hutton WC: Adjacent segment motion after a simulated lumbar fusion in different sagittal alignments: a biomechanical analysis. Spine 28: 1560-1566, 2003

2) Brantigan JW, Steffee AS, Lewis ML, Quinn LM, Persenaire JM: Lumbar interbody fusion using Brantigan $\mathrm{I} / \mathrm{F}$ cage for posterior lumbar interbody fusion and the variable pedicle screw placement system: two-year results from a Food and Drug Administration investigational device exemption clinical trial. Spine 25: 1437-1446, 2000

3) Burns BH: An operation for spondylolisthesis. Lancet 1: 1233-1239, 1933

4) Duggal N, Mendiondo I, Jhawar BS, Pares HR, Das K, Kenny KJ, Dickman CA: Anterior lumbar interbody fusion for treatment of failed back surgery syndrome: an outcome analysis. Neurosurgery 54: 636-644, 2004

5) Fairbank J, Couper J, Davies J, O’Brien JP: The Oswestry low back pain disability questionnaire. Physiotherapy 66: 271-273, 1980

6) Herkowitz HN, Garfin SR, Balderston RA, Eismont FJ, Bell GR, Wiesel SW: Rothman-Simeone The Spine, 
ed 4. Philadelphia, WB Saunders, 1999, pp 1089-1093

7) Hosono N, Namekata M, Makino T, Miwa T, Kaito T, Kaneko N, Fuji T: Perioperative complications of primary posterior lumbar interbody fusion for nonisthmic spondylolisthesis: analysis f risk factors. J Neurosurg Spine 9: 403-407, 2008

8) Humphreys SC, Hodges SD, Patwardhan AG, Eck JC, Murphy RB, Covington LA: Comparison of posterior and transforaminal approaches to lumbar interbody fusion. Spine 26: 567-571, 2001

9) Ishihara $H$, Osada $R$, Kanamori M, Kawaguchi $Y$, Ohmori K, Kimura T, Matsui H, Tsuji H: Minimum 10-year follow-up study of anterior lumbar interbody fusion for isthmic spondylolisthesis. J Spinal Disord 14: 91-99, 2001

10) Jang JS, Lee SH: Clinical analysis of percutaneous facet screw fixation after anterior lumbar interbody fusion. J Neurosurg Spine 3: 40-46, 2005

11) Jang JS, Lee SH: Minimally invasive transforaminal lumbar interbody fusion with ipsilateral pedicle screw and contralateral facet screw fixation. $J \mathrm{Neu}-$ rosurg Spine 3: 218-223, 2005

12) Kai Y, Oyama M, Morooka M: Posterior lumbar interbody fusion using local facet joint autograft and pedicle screw fixation. Spine 29: 41-46, 2004

13) Kulkarni SS, Lowery GL, Ross RE, Ravi Sankar K, Lykomitros V: Arterial complications following anterior lumbar interbody fusion: report of eight cases. Eur Spine J 12: 48-54, 2003

14) Kwon BK, Albert TJ: Adult low-grade acquired spondylolytic spondylolisthesis. Evaluation and management. Spine 30: S35-S41, 2005

15) Lee DY, Jung TG, Lee SH: Single-level instrumented mini-open transforaminal lumbar interbody fusion in elderly patients. J Neurosurg Spine 9: 137-144, 2008

16) Lee DY, Lee SH, Kim SK, Maeng DH, Jang JS: A morphometric analysis of neuroforamen in grade I isthmic spondylolisthesis by anterior lumbar interbody fusion with pedicle screw fixation. J Korean Neurosurg Soc 41: 377-381, 2007

17) Lee DY, Lee SH, Maeng DH, Jang JS: Isthmic spondylolisthesis associated with foraminal disc herniation treated by anterior lumbar interbody fusion. $J$ Korean Neurosurg Soc 38: 320-322, 2005

18) Lee SH, Choi WG, Lim SR, Kang HY, Shin SW: Minimally invasive anterior lumbar interbody fusion followed by percutaneous pedicle screw fixation for isthmic spondylolisthesis. Spine J 4: 644-649, 2004

19) Lee SH, Kang BU, Jeon SH, Park JD, Maeng DH, Choi YG, Choi WC: Revision surgery of the lumbar spine: anterior lumbar interbody fusion followed by percutaneous pedicle screw fixation. J Neurosurg Spine 5: 228-233, 2006

20) Min JH, Jang JS, Lee SH: Comparison of anterior- and posterior-approach instrumented lumbar interbody fusion for spondylolisthesis. J Neurosurg Spine 7: 21-26, 2007

21) Miyakoshi N, Abe E, Shimada Y, Okuyama K, Suzuki T, Sato K: Outcome of one-level posterior lumbar in- terbody fusion for spondylolisthesis and postoperative intervertebral disc degeneration adjacent to the fusion. Spine 25: 1837-1842, 2000

22) Newman MH, Grinstead GL: Anterior lumbar interbody fusion for internal disc disruption. Spine 17: 831-833, 1992

23) Okuda S, Oda T, Miyauchi A, Haku T, Yamamoto T, Iwasaki M: Surgical outcomes of posterior lumbar interbody fusion in elderly patients. J Bone Joint Surg Am 88: 2714-2720, 2006

24) Okuyama K, Abe E, Suzuki T, Tamura Y, Chiba M, Sato K: Posterior lumbar interbody fusion: a retrospective study of complications after facet joint excision and pedicle screw fixation in 148 cases. Acta Orthop Scand 70: 329-334, 1999

25) Penta M, Fraser RD: Anterior lumbar interbody fusion. A minimum 10-year follow-up. Spine 22: 2429-2434, 1997

26) Scaduto AA, Gamradt SC, Yu WD, Huang J, Delamarter RB, Wang JC: Perioperative complications of threaded cylindrical lumbar interbody fusion devices: anterior versus posterior approach. J Spinal Disord Tech 16: 502-507, 2003

27) Shim CS, Lee SH, Shin HD, Kang HS, Choi WC, Jung B, Choi G, Ahn Y, Lee S, Lee HY: CHARITE versus ProDisc: a comparative study of a minimum 3-year follow-up. Spine 32: 1012-1018, 2007

28) Umehara S, Zindrick MR, Patwardhan AG, Havey RM, Vrbos LA, Knight GW, Miyano S, Kirincic M, Kaneda K, Lorenz MA: The biomechanical effect of postoperative hypolordosis in instrumented lumbar fusion on instrumented and adjacent spinal segments. Spine 25: 1617-1624, 2000

Address reprint requests to: Dong Yeob Lee, M.D., Department of Neurosurgery, Wooridul Spine Hospital, 47-4 Chungdam-dong Gangnam-gu, Seoul 135-100, R.O.K.

e-mail:nsdrlee@naver.com

\section{Commentary}

In this study, Lee and colleagues demonstrate that two level anterior lumbar interbody fusion (ALIF) augmented with percutaneous posterior segmental instrumentation with pedicle screws and rods is a safe and effective option for the treatment of two level lumbar instability, i.e. spondylolisthesis. 24 consecutive patients ( 8 males and 16 females) with back pain, leg pain, and/or neurogenic claudication and imaging studies demonstrating two consecutive levels of lumbar instability were treated at the Wooridul Spine Hospital utilizing this strategy. They were analyzed in a retrospective fashion both clinically and radiographically. Clinical measures assessed included the visual analog scale (VAS) and the Oswestry 
Disability Index (ODI). Diagnostic imaging was used to assess lumbar sagittal alignment, bony fusion, and adjacent segment degeneration.

Patients (average age 56.3 years) were followed for an average duration of 39.4 months. Clinical success, defined as $a \geq 2$ point improvement in the VAS, and a $\geq 25 \%$ improvement in ODI and no major complication related to the surgery, was achieved in 22 of the 24 patients. VAS for back pain decreased from an average of 6.5 preoperatively to 3.0 postoperatively ( $p$ $<0.001)$. VAS for leg pain improved from 6.8 preoperatively to 1.9 postoperatively $(p<0.001)$. Average ODI improved from $46.9 \%$ to $16.3 \%$ after surgery ( $p<0.001$ ). Radiographically, segmental lordosis, whole lumbar lordosis, and sacral tilt all increased significantly with surgery. Radiographic signs of bony fusion were observed in 21 of 24 patients. Three patients were considered to have nonunion and 2 patients had posterior hardware failure with screw fracture. None of these were considered symptomatic and no patient underwent revision. Evidence of cephalad adjacent segment degeneration was observed in 8 of the 24 patients with 7 patients demonstrating $a>10 \%$ loss of disk height and 1 patient showing angular instability. None of these patients had symptoms related to their adjacent segment disease, and no revision surgeries were performed.

This study demonstrates that ALIF augmented with percutaneous posterior segmental stabilization is a powerful tool for the treatment of lumbar instability with good clinical and radiographic results. The benefit of superior anterior column support and minimal posterior disruption achieved using this strategy must be weighed against the potential complications of an anterior retroperitoneal approach to the lumbar spine. Larger multicenter prospective studies are needed before conclusion can be made regarding the superiority of this approach versus an all posterior approach for the treatment of lumbar instability.

Joshua E. Heller, M.D. and Christopher M. LOFTUS, M.D., F.A.C.S.* Departments of Orthopaedic Surgery and Neurosurgery University of Virginia Health System Charlottesville, Virginia, U.S.A.; ${ }^{*}$ Department of Neurosurgery Temple University School of Medicine Philadelphia, Pennsylvania, U.S.A. 